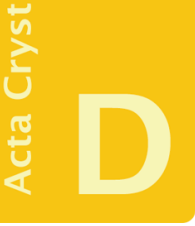

Volume 71 (2015)

Supporting information for article:

Structure of Csd3 from Helicobacter pylori, a cell-shape determining metallopeptidase

Doo Ri An, Hyoun Sook Kim, Jieun Kim, Ha Na Im, Hye Jin Yoon, Ji Young Yoon, Jun Young Jang, Dusan Hesek, Mijoon Lee, Shahriar Mobashery, SoonJong Kim, Byung II Lee and Se Won Suh 


\begin{tabular}{lcc}
\hline \multicolumn{3}{l}{ Table S1. Statistics on data collection of Zn SAD data sets } \\
\hline Data set & Zn SAD (Form 1) & Zn SAD (Form 2) \\
Space group & $P 2{ }_{1} 2_{1}{ }_{1}$ & $P 6522$ \\
Unit cell lengths, a, b, c $(\AA)$ & $62.2,113.0,112.7$ & $92.4,92.4,186.7$ \\
Unit cell angles, $\alpha, \beta, \gamma\left({ }^{\circ}\right)$ & $90,90,90$ & $90,90,120$ \\
X-ray wavelength $(\AA)$ & 1.2820 & 1.2820 \\
Resolution range $(\AA)$ & $50.0-2.30(2.34-2.30)^{a}$ & $50.0-2.35(2.39-2.35)^{a}$ \\
Total / unique reflections & $559,489 / 68,296^{b}$ & $544,542 / 36,489^{b}$ \\
Completeness $(\%)$ & $100.0(99.6)^{a, b}$ & $99.1(97.4)^{a, b}$ \\
$<_{\perp>}{ }^{c}<\sigma_{I}>$ & $57.2(3.6)^{a, b}$ & $65.4(8.7)^{a, b}$ \\
$R_{\text {merge }}{ }^{a}(\%)$ & $8.8(83.9)^{a, b}$ & $14.4(72.4)^{a, b}$ \\
$C_{1 / 2}{ }^{a}(\%)$ & $99.8(85.3)^{a}$ & $99.5(86.5)^{a}$ \\
\hline
\end{tabular}

${ }^{a}$ Values in parentheses refer to the highest resolution shell.

${ }^{b}$ Friedel pairs were treated as separate observations.

${ }^{c} R_{\text {merge }}=\Sigma_{\mathrm{h}} \Sigma_{\mathrm{i}}\left|I(h)_{\mathrm{i}}-<I(h)>\right| / \Sigma_{\mathrm{h}} \Sigma_{\mathrm{i}} I(h)_{\mathrm{i}}$, where $I(h)$ is the intensity of reflection $h, \Sigma_{\mathrm{h}}$ is the sum over all reflections, and $\Sigma_{\mathrm{i}}$ is the sum over i measurements of reflection $h$.

${ }^{d} \mathrm{CC}_{1 / 2}$ is the correlation coefficient of the mean intensities between two random half-set of data. 


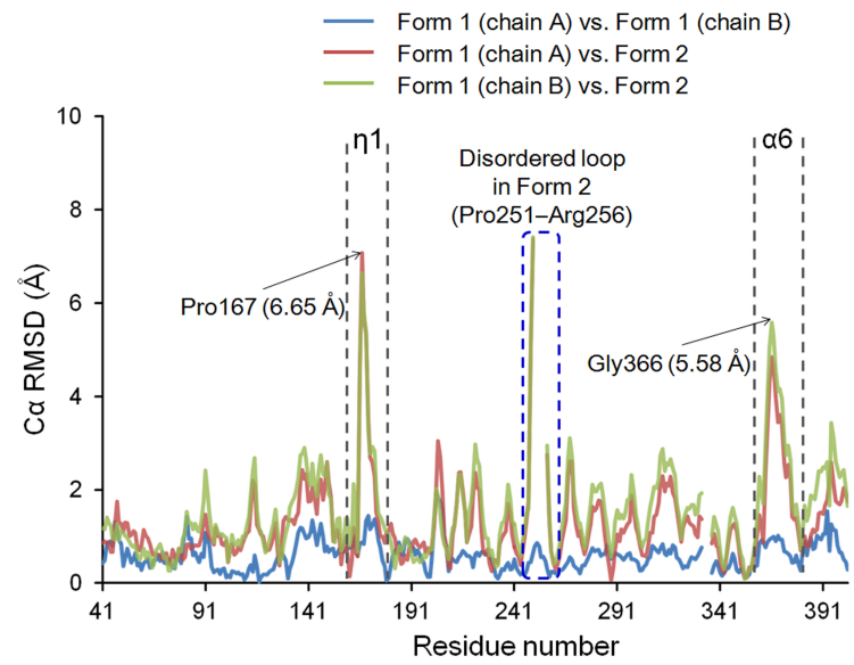

(a)

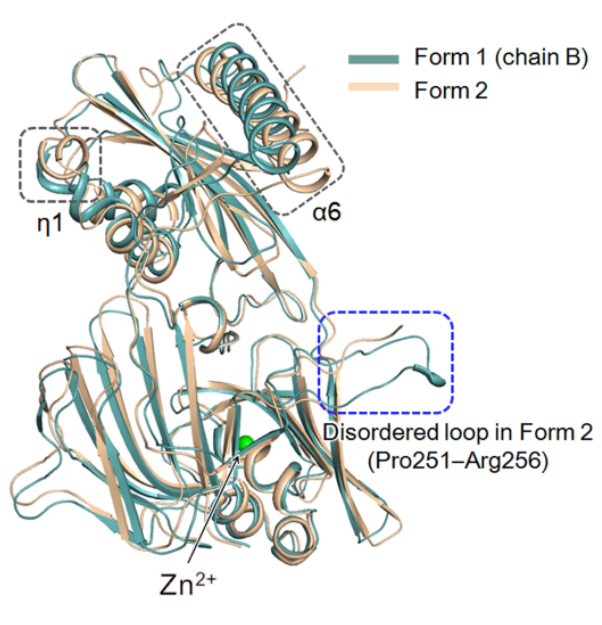

(b)

\section{Supplementary Figure S1}

Comparisons of $\mathrm{Csd} 3_{\Delta 41}$ monomer structures. (a) Plot of $\mathrm{C} \alpha$ r.m.s. deviations for pairwise comparisons among three monomer models of $\mathrm{Csd}_{\Delta 41}$. Chains $\mathrm{A}$ and $\mathrm{B}$ of Form 1 crystal are more similar to each other than they are to Form 2 crystal. The $\eta 1$ and $\alpha 6$ helices show large deviations between Form 1 and Form 2 crystals. (b) A superposition of two monomer models of Csd $3_{\Delta 41}$. Models of Form 1 crystal (chain B) and Form 2 crystal are colored in teal blue and light orange, respectively. The $\eta 1$ and $\alpha 6$ helices, which show significant conformational differences, are highlighted by gray dotted boxes. Residues Pro251-Arg256 are disordered in Form 2 crystal and are highlighted by a blue dotted box. 


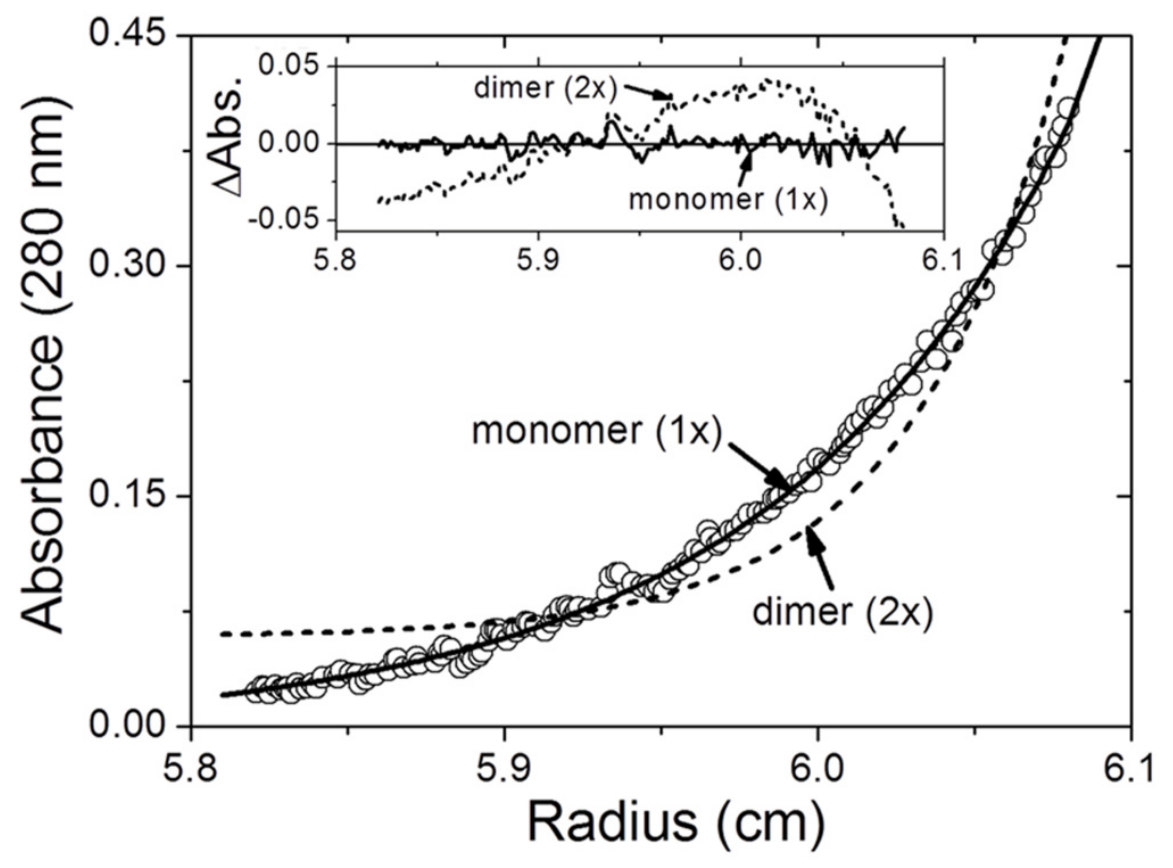

\section{Supplementary Figure S2}

Sedimentation equilibrium distributions of $H$. pylori $\operatorname{Csd} 3_{\Delta 41}$. Representative data measured at $18,000 \mathrm{rpm}$ using the $3.77 \mu \mathrm{M}$ protein concentration are shown. The circles are experimental absorbance data at $280 \mathrm{~nm}$ and the solid line is a fitting line for a homogeneous monomer $(1 \times)$ model. The dotted line is a fitting line for an ideal homogeneous dimer $(2 \times)$ model. (Inset) Distributions of the residuals for monomer $(1 \times$, solid line) and dimer $(2 \times$, dotted line) models, respectively. Random distribution of the residuals for the monomer $(1 \times)$ model indicates that $\mathrm{Csd} 3_{\Delta 41}$ exists as homogeneous monomers in solution. 


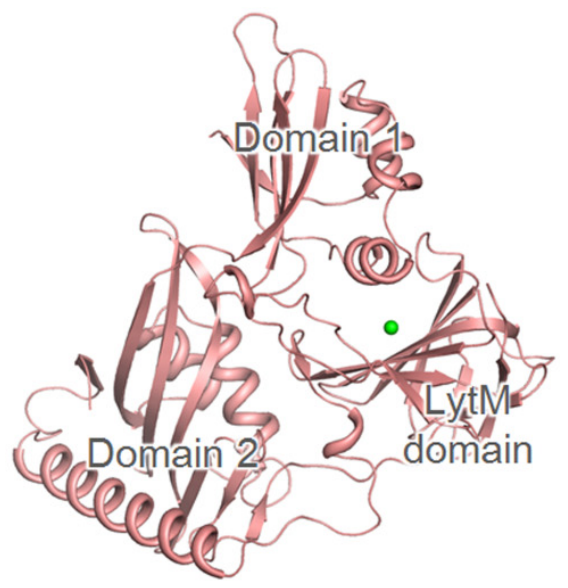

H. pylori Csd3

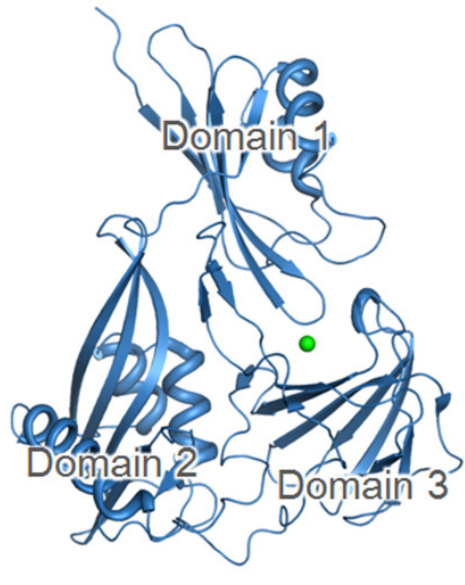

NMB0315

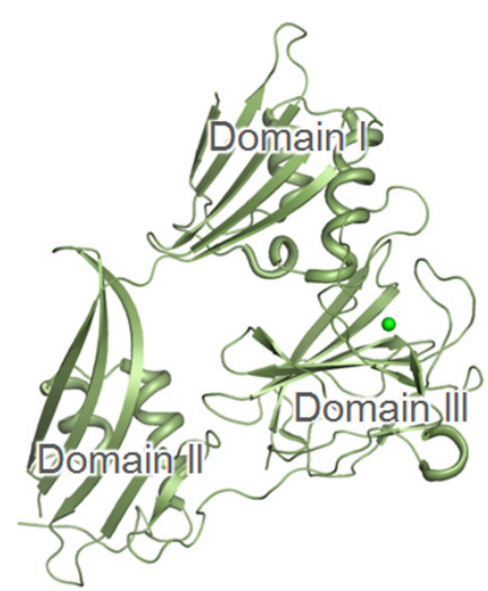

VC0503

\section{Supplementary Figure S3}

Ribbon diagram of three-domain proteins showing structural similarity to the entire structure of H. pylori $\operatorname{Csd} 3_{\Delta 41}$. The structure of H. pylori $\operatorname{Csd} 3_{\Delta 41}$ (deep salmon) is on the left, $N$. meningitides NMB0315 (sky blue; PDB code, 3SLU) in the middle, and V. cholera VC0503 (pale green; PDB code, 2GU1) on the right. Metal ions are shown as green spheres. In NMB0315, the $\mathrm{Zn}^{2+}$ ion was replaced with a $\mathrm{Ni}^{2+}$ ion during affinity chromatography (Wang et al., 2011). 
Domain 1

50

60

70

80

.....ER ... LVWDKLTLLGFLEKNH IP ... QKLY . . YNLSSQDKELSAEIQSNVTY . .

Domain 2 IFTRKEKTLLLSLQTSPYQDIIKATNDPLLANQLMNAYKKSVPFKRLVKNDKIAIVYTRD

Domain 2

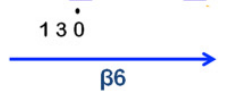

${ }^{140^{\circ}}$ eleeele $^{15}$ $50 \quad 160^{\circ}$

170
$l 1$

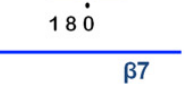

Domain 1
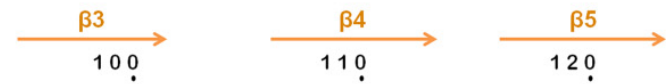

Domain 1 YTLRDAN. NTLIQALIPISQDLQIHIYKKGEDYFLDFIPI . . .

Domain 2 YRVGQAFGQPTIKMAMVSSRSNQYYLFSHSNGHYYDSKAQEVAG

Domain 2 $\rightarrow \frac{19 \dot{0}}{\beta 8} 20 \dot{0} \quad \frac{21 \dot{0}}{\beta 9} \quad \underset{\beta 10}{\longrightarrow} \quad \underset{\beta 11}{\longrightarrow}$

(a)
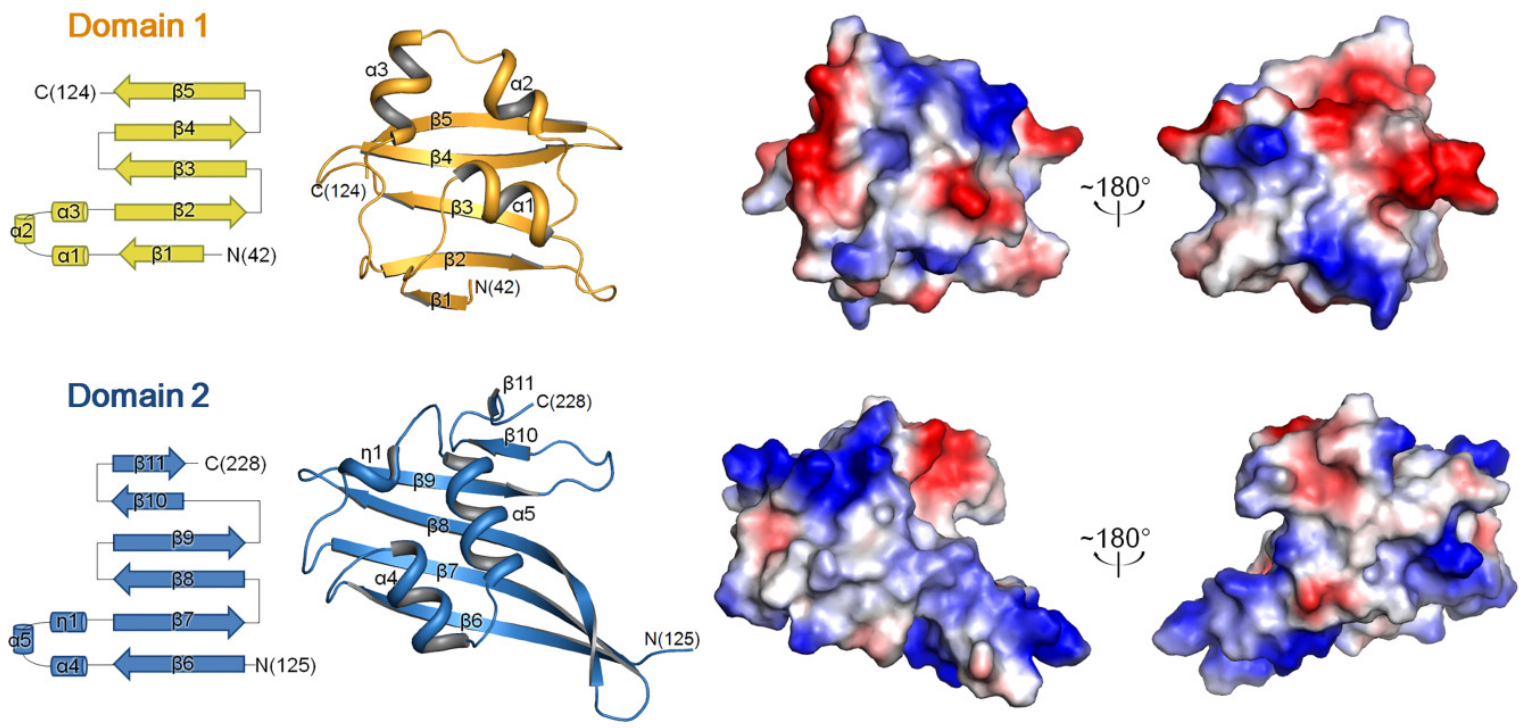

(b)

\section{Supplementary Figure S4}

Sequence and structure comparison of Csd3 domain 1 and domain 2. (a) Sequence alignment of Csd3 domain 1 and domain 2 with secondary structures. (b) Comparison of charge distributions on the surface of $\mathrm{Csd} 3$ domain 1 (top) and domain 2 (bottom). Topology and ribbon diagrams for domain 1 and domain 2 are colored as in Fig. 1a, with the secondary structure elements labeled. Two different views of the electrostatic potential surface diagrams related by a $180^{\circ}$ rotation are shown next to the ribbon diagrams. 


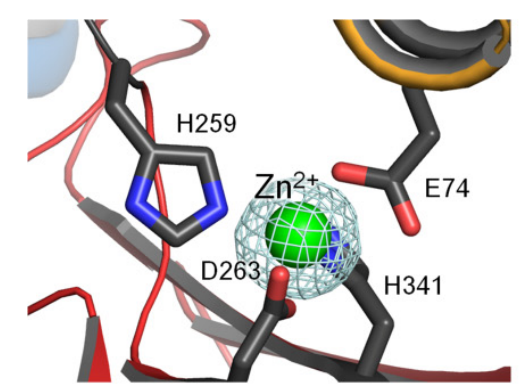

Form 1 (chain A)

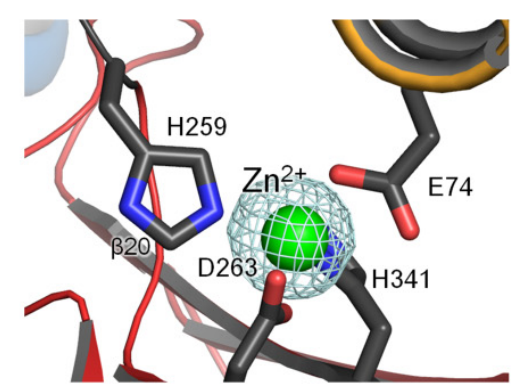

Form 1 (chain B)

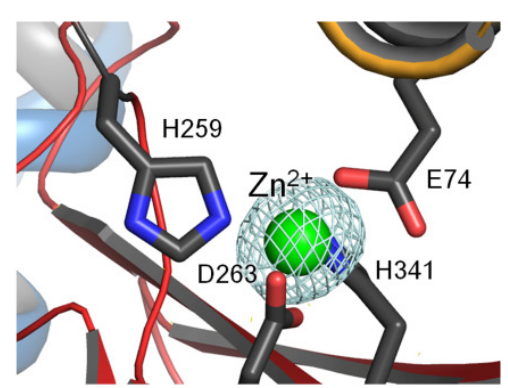

Form 2

\section{Supplementary Figure S5}

Electron density of metal ions bound to the $\mathrm{Zn}^{2+}$-binding site in the anomalous difference Fourier maps (contoured at $10 \sigma$ and colored in cyan), calculated using the Zn SAD data sets from Form 1 and Form 2 crystals (Supplementary Table S1). Ribbon diagrams are colored as in Fig. $1 a$.

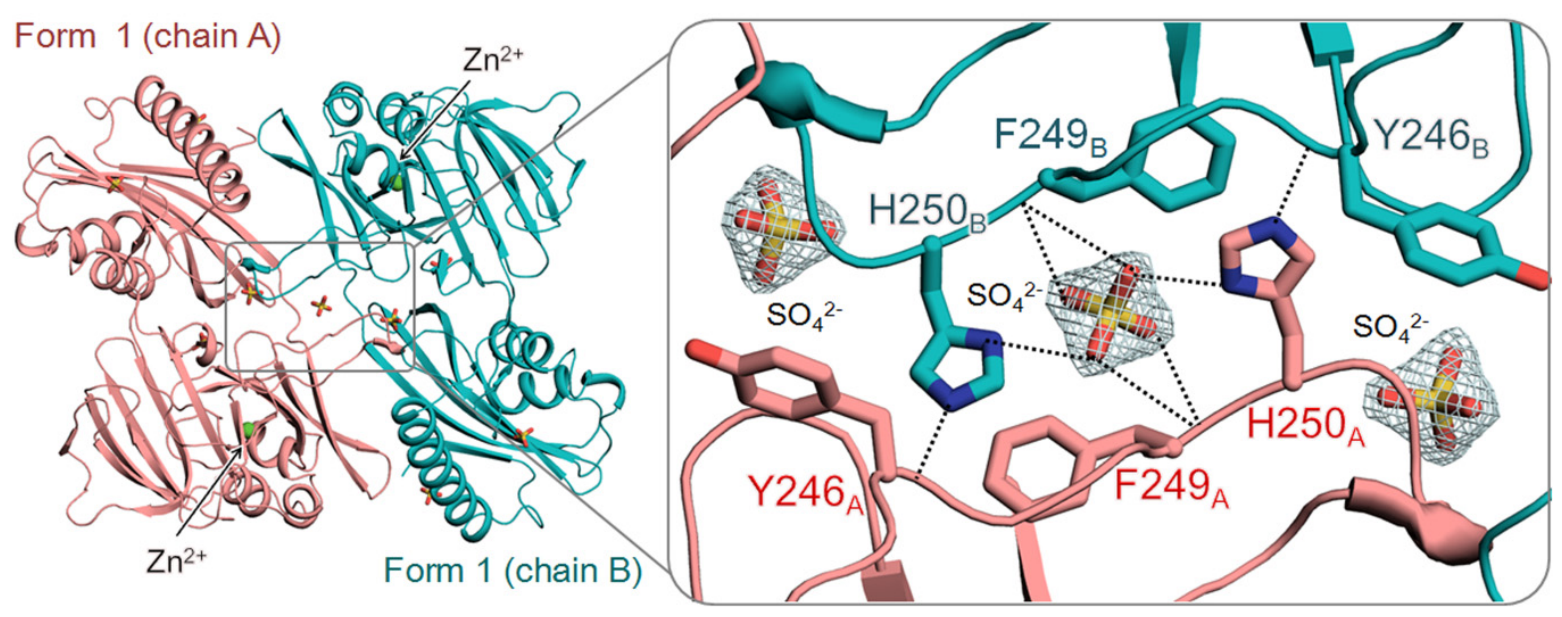

\section{Supplementary Figure S6}

Crystal packing interactions in Form 1 crystal of H. pylori Csd $3_{\Delta 41}$. Chain A (salmon) and chain $\mathrm{B}$ (teal blue) of $\mathrm{Csd}_{\Delta 41}$ in the asymmetric unit are shown in ribbon diagrams. $\mathrm{Zn}^{2+}$ ions are indicated by green spheres. Nine sulfate ions are shown in stick models. A close-up view on the right represents inter-chain interactions mediated by a sulfate ion at the noncrystallographic two-fold symmetry axis. Residues at the interface between two chains are shown in stick models and labeled. The electron density for the sulfate ions in the $2 m F_{o}-$ $D F_{c}$ map are shown in cyan colored mesh (contoured at $2 \sigma$ ). Hydrogen bonds and salt bridges are indicated by black dotted lines. 


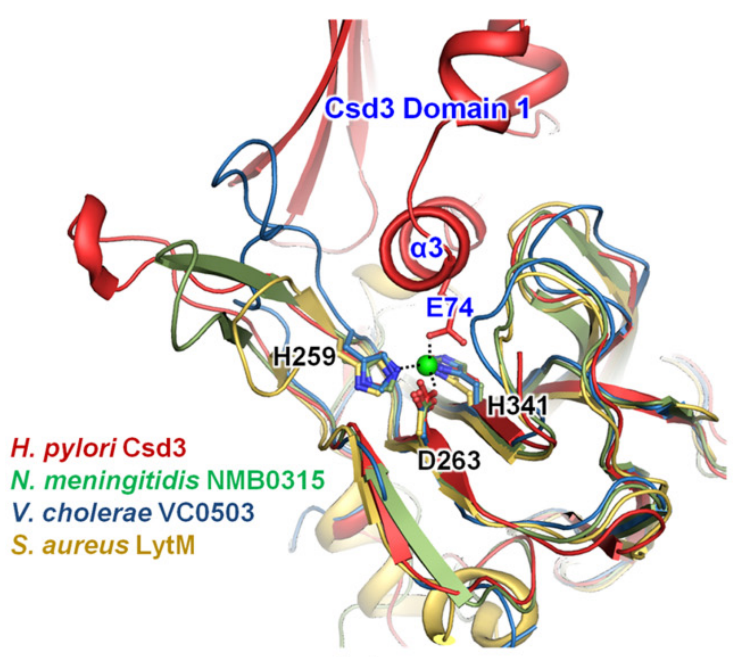

(a)

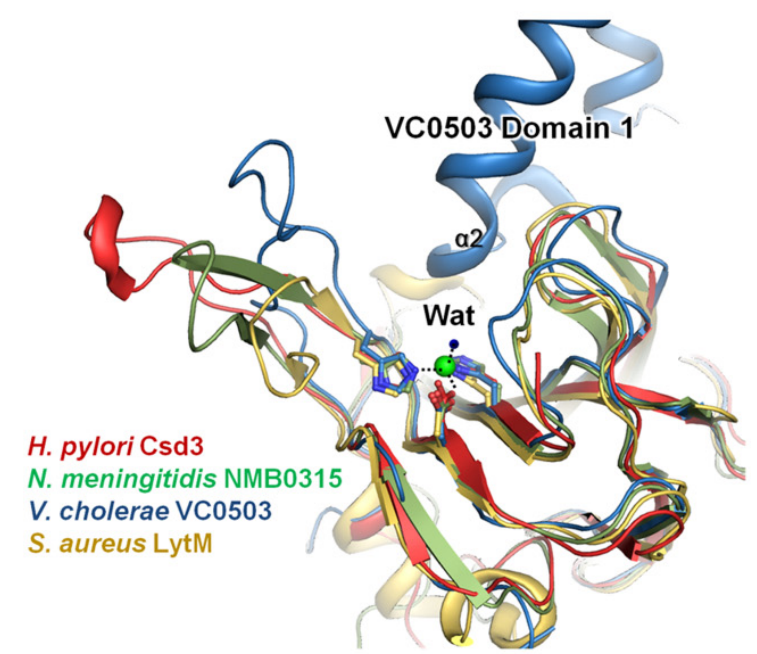

(c)

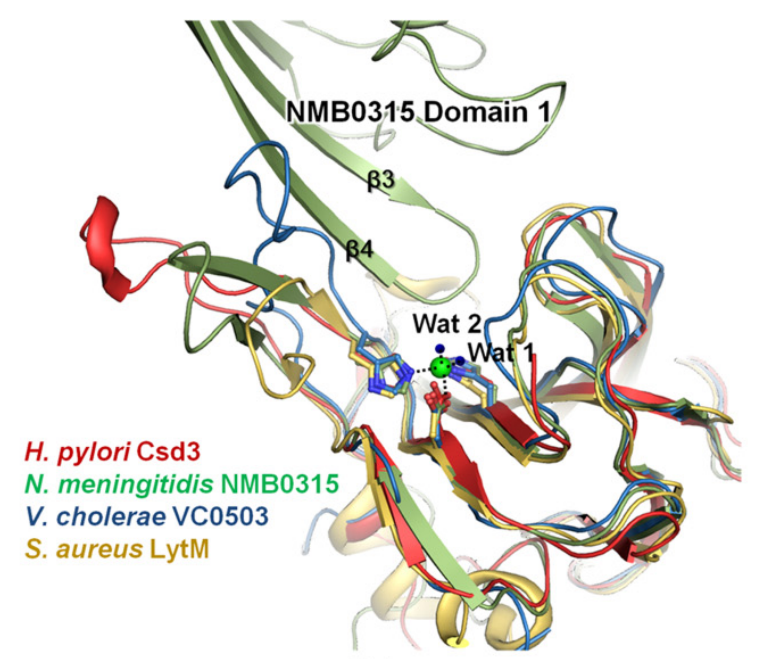

(b)

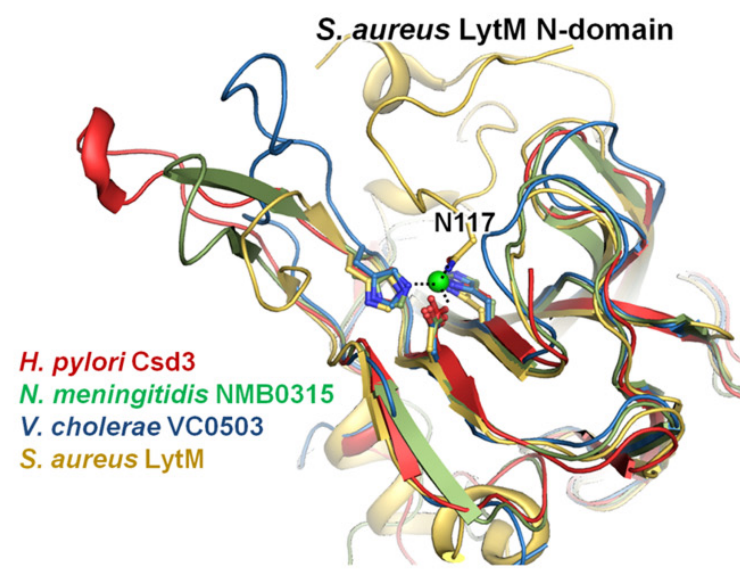

(d)

\section{Supplementary Figure S7}

Structural superposition of LytM domains of M23B family metallopeptidases in the inhibited state. Four LytM domains are superimposed as in Fig. $4 b$ but are shown in a different orientation. $(a-d)$ Domain 1 of $H$. pylori $\operatorname{Csd} 3(a)$, and corresponding inhibitory domains of $N$. meningitidis NMB0315 (b), V. cholerae VC0503 (c), and S. aureus LytM (d) are shown in addition to the superposed LytM domains. Metal ions are shown as green spheres. In $\mathrm{NMB} 0315$, the $\mathrm{Zn}^{2+}$ ion was replaced with a $\mathrm{Ni}^{2+}$ ion during affinity chromatography (Wang et al., 2011). Water molecules are shown as blue dots. Black dotted lines denote metal coordination.

\section{Reference}

Wang, X., Yang, X., Yang, C., Wu, Z., Xu, H. \& Shen, Y. (2011). PLoS One 6, e26845. 